\title{
EDIFICE TROPHIQUE PARTIEL DU COURS SUPERIEUR D'UN RUISSEAU FORESTIER
}

\author{
par B. DUMONT ${ }^{1}$ et J. VERNEAUX ${ }^{2}$.
}

Un échantillonnage du peuplement ichtyologique (octobre 1972) et trois séries de prélèvements d'invertébrés (janvier, mars et mai 1973) effectués dans le cours supérieur d'un ruisseau forestier, ont fait l'objet d'une analyse des relations trophiques à partir de l'étude du régime alimentaire de la plupart des 57 espèces répertoriées.

Les différentes variations observées (modifications du régime alimentaire en fonction de l'âge, de la taille, de l'époque et des habitats), ainsi que le schéma général de l'édifice biologique, soulignent la complexité des relations trophiques à l'intérieur de ce peuplement proche des sources et de composition relativement simple.

\section{Some trophic relationships in the upper reaches of a forest stream.}

One sample of the fish population (october 1972) and three series of samples of invertebrates (January, March and May 1973) were taken in the upper part of a forest brook.

Trophic relationships were analysed from a study of the feeding habits of 57 species.

The modification of the feeding habits with age, length, time and habitat, and also the general picture of the biological structure, emphasize the complexity of the trophic relationships within this headwater stream with its simple community.

\section{1. - INTRODUCTION}

Si les relations trophiques existant au sein des communautés aquatiques ont, depuis longtemps, fait l'objet d'études relativement nombreuses, il convient de remarquer à la suite de MANN (1969) que les travaux ont souvent porté sur des «espèces solitaires ou des chaînes alimentaires isolées ". (SLACK 1936; HaNNA 1957; JoNES 1950 ; Chapman et Demory 1963 ; Griffiths 1970 ; Jenkins, Feldmeth et Elliott 1970 ; Richardoson et Gaufin 1971 ; SiefFert 1972; LadLe,

1. D'après les résultats d'un travail de D.E.A. soutenu le 28.06 .73 à l'Université Claude-Bernard, Lyon 1.

2. Lab. Hydroécol., Div. Qual. Eaux, P., Pisc., C.T.G.R.E.F. Paris et Centre Hydrobiol. Univ. Besançon 
Bass et Jenkins 1972 ; Kolucher et Mrnshall 1973; Madsen 1974).

Le présent travail effectué sur le cours supérieur d'un ruisseau forestier fait partie d'un ensemble de recherches visant à définir les mécanismes de l'organisation typologique de l'écosystème « eau courante » (VeRnEAUX, 1973) et des phénomènes appréhendés. La première phase de cette approche traite des sources de nourriture ainsi que des modalités de l'alimentation des différentes espèces rencontrées; les régimes alimentaires, les modes de vie et leurs variations ont ainsi été examinés pour une cinquantaine d'espèces.

Les premiers résultats obtenus permettent de proposer une esquisse de l'édifice trophique représentatif du cours supérieur d'un ruisseau de faibles dimensions $(1<1<4 \mathrm{~m})$ au peuplement relativement simple.

\section{2. -- LE MILIEU}

Le secteur prospecté est situé sur le cours des Doulonnes, ruisseau de la bordure septentrionale de la forêt de Chaux, dont le peuplement appartient au Biocénotype B2, selon VeRnEAUX (1973) associé à l'Hypocrenon [Illies (1963)]. Ce secteur est caractérisé par une pente relativement faible $\left(7 \%\right.$, des eaux fraîches $\left(9<0<13^{\circ} \mathrm{C}\right)$, limpides, peu minéralisées $\left(C \leqslant 120 \mu \mathrm{mhos} / \mathrm{cm} / \mathrm{cm}^{2}\right)$, la présence dominante de galets siliceux et de dépôts sédimentaires humiques dûs à l'environnement forestier (VERNEAUX, 1968).

\section{3. - METHODẼS UTILISEES ET EXPRESSION DES RESULTATS}

L'inventaire des macroinvertébrés benthiques peuplant les différents habitats du secteur a été effectué au cours de trois campagnes de prélèvements (janvier, mars, mai 1973), au moyen d'un filet échantillonneur standard de $1 / 10 \mathrm{~m}^{2}$ et d'une sonde verticale de $2 \mathrm{dm}^{3}$. La faune était immédiatement fixée (formol $5 \%$ ) afin d'éviter toute prédation ultérieure à la capture.

L'analyse de l'échantillon comprenait le dénombrement des invertébrés présents, leur mesure et l'examen des contenus digestifs ; selon l'abondance de l'espèce, une plus ou moins grande partie de la population fut examinée.

Les poissons, peu abondants à ce niveau, ont été échantillonnés au moyen d'un appareil standard de capture à courant continu.

En raison de la pisciculture pratiquée dans le cours d'eau ( «uisseau pépinière ") où cohabitent Truitelles, Chabots et Lamproies, 
seules les deux dernières espèces ont été retenues pour l'analyse trophique.

- Détritivores et consommateur primaires.

Les observations effectuées conduisent à distinguer cinq catégories d'aliments :

- les débris végétaux altérés ou ligneux,

- les débris végétaux récents,

- les diatomées,

- les stades Chantransia (Protonema d'une algue rouge du genre Batrachospermun FELDAM 1963),

Les algues non citées précédemment, souvent uni ou paucicellulaires (Desmidiales, Tétrasporales).

- Espèces carnassières.

Les résultats sont exprimés par quatre données :

- la composition globale de la faune, exprimée en pourcentage du nombre total d'individus capturés,

- la répartition des proies au sein de chaque espèce exprimée en pourcentage du nombre total de proies ingérées,

- une éventuelle "sélection apparente" (ALLEN 1941; Jones 1950 ; Hunt et JoNEs 1972), ne permettant cependant pas de faire la part entre le choix du prédateur et la disponibilité de la proie, déterminée à l'aide du coefficient :

$$
s=\frac{\text { pourcentage de la proie dans les contenus digestifs }}{\text { pourcentage de la proie dans le benthos }}
$$

- l'intensité de prédation, ou nombre de proies ingérées par 100 prédateurs, calculée dans le cas du Chabot à partir de classes de taille choisies arbitrairement de 10 en $10 \mathrm{~mm}$.

\section{4. - RESULTATS}

\section{1. - Principaux régimes alimentaires (tableau I).}

- la majorité des espèces appartient au groupe des "détritivores herbivores » possédant sensiblement le même régime alimentaire de base ;

- 9 espèces «microphytophages » constituent le groupe des consommateurs primaires stricts,

- le groupe des "omnivores" comprend 6 espèces qui ingèrent simultanément des élément végétaux et animaux (invertébrés), 
Tableau I. - Composition des principaux groupes de consommateurs et abondance relative des différentes catégories alimentaires dans l'alimentation des espèces inventoriées. Trois classes d'abondance ont été utilisées :

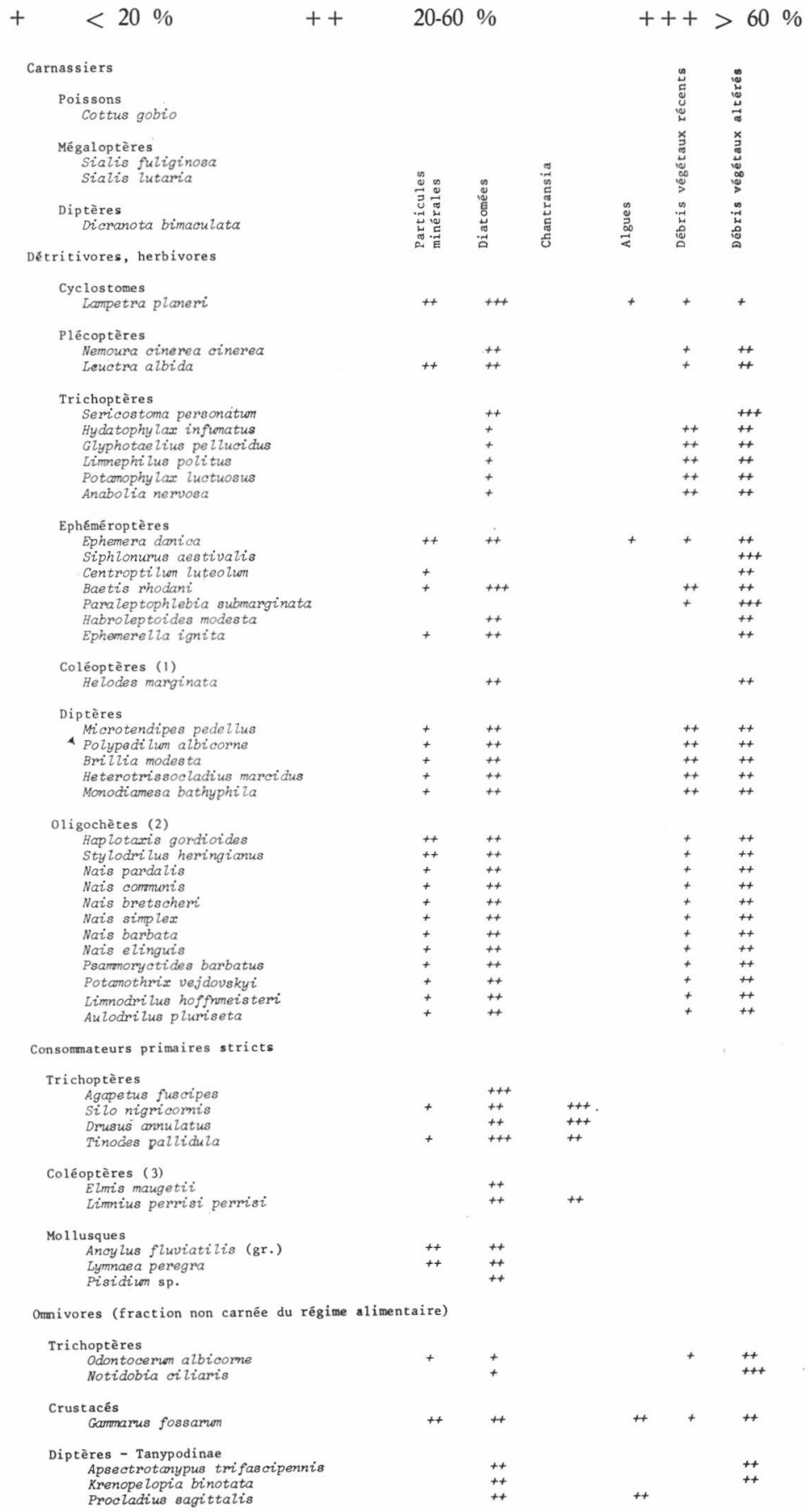

(1) Espèces déterminées par J.P. Vergon, Labo. Hydroécol., Div. Qualité des Eaux, P. Pisc., C.T.G.R.E.F., Centre Hydobiol., Besançon.

(2) Espèces déterminées par M. Lafont, Centre Hydrobiol., Besançon.

(3) Espèces déterminées par A.L. Roux, Départ. Biol. Anim., Univ. Claude-Bernard, Lyon. 
- le tableau II donne la composition de la fraction carnée du régime alimentaire des espèces omnivores. On y relève quelques cas de cannibalisme.

Tableau II. - Fraction carnée du régime alimentaire des espèces omnivores exprimée en $\%$ du nombre total de proies capturées par chaque espèce.

\begin{tabular}{|c|c|c|c|c|c|}
\hline & $\begin{array}{c}O . \\
\text { albicorne }\end{array}$ & $\begin{array}{c}G . \\
\text { fossarum }\end{array}$ & $\begin{array}{l}A . \\
\text { trifascipennis }\end{array}$ & $\begin{array}{c}P . \\
\text { sagittalis }\end{array}$ & $\begin{array}{c}K . \\
\text { binotata }\end{array}$ \\
\hline \multicolumn{6}{|l|}{$\%$ des carnivores } \\
\hline dans la population & 80 & 50 & 50 & 50 & 28 \\
\hline Trichoptères & 46 & 7 & - & 一 & 一 \\
\hline Epheméroptères & 39 & 57 & - & - & - \\
\hline Diptères & 15 & 20 & 41 & 50 & 65 \\
\hline Ostracodes & - & - & 24 & 30 & - \\
\hline Copépodes & - & - & - & 10 & 35 \\
\hline Cannibalisme & - & 6 & 35 & 10 & - \\
\hline
\end{tabular}

N.B. Notidobia ciliaris présentant seulement deux cas de prédation (2 C. luteolum, et 5 Ostracodes), ne figure pas dans ce tableau.

- Dans le tableau III figure la répartition des proies composant l'alimentation des espèces carnassières.

TABlEAU III. - Régime alimentaire des espèces carnassières (pourcentages).

\begin{tabular}{|c|c|c|c|c|c|}
\hline & $\underset{\text { gobio }}{C .}$ & $\begin{array}{c}R . \\
\text { septentrionis }\end{array}$ & $\begin{array}{c}D . \\
\text { bimaculata }\end{array}$ & $\begin{array}{c}S . \\
\text { fuliginosa }\end{array}$ & $\begin{array}{c}S . \\
\text { lutaria }\end{array}$ \\
\hline Mégaloptères & 2 & - & - & - & 一 \\
\hline Gammares & 35 & - & 一 & 40 & - \\
\hline Trichoptères & 7 & 55 & $\longrightarrow$ & 30 & - \\
\hline Ephéméroptères & 22 & 45 & 45 & 16 & - \\
\hline Dipteres & 32 & - & 43 & 14 & - \\
\hline Ostracodes & 2 & 一 & - & - & - \\
\hline Copépodes & - & - & 12 & - & - \\
\hline Oligochètes & - & - & - & - & 100 \\
\hline
\end{tabular}

La différence des régimes alimentaires de $S$. lutaria et $S$. fuliginosa est à mettre en relation avec leurs habitats respectifs : généralement les dépôts humiques en facies lénitique pour le premier, les galets et les graviers en courant faible pour le second.

\section{2. - Les coefficients de sélection chez les espèces carnassiè- res.}

Exemple : Cottus gobio (tableau IV).

Une consommation préférentielle très nette apparaît pour Sialis fuliginosa et les Ephéméroptères, ce dernier groupe étant composé à $98 \%$ de deux espèces du genre Baetis : $B$. rhodani et $B$. muticus. 
Tableau IV. - Coefficient de sélection apparente chez Cottus gobio L.

\begin{tabular}{ll}
\hline Mégaloptères & 9.10 \\
Ephéméroptères & 4.30 \\
Diptères & 1.40 \\
Trichoptères & 0.94 \\
Gammares & 0.70 \\
Coléoptères & 0.14 \\
\hline
\end{tabular}

Exemple : les invertébrés carnassiers (tableau V).

L'analyse porte sur l'alimentation globale d'une centaine d'individus appartenant aux dix espèces mentionnées dans les tableaux II et III. La sélection apparente est marquée pour les Ephéméroptères et les Trichoptères.

Tableau V. - Coefficients de sélection apparente pour l'ensemble de 10 espèces d'invertébrés carnassiers.

\begin{tabular}{ll}
\hline Ephéméroptères & 6.70 \\
Trichoptères & 2.70 \\
Diptères & 1.20 \\
Coléoptères & 0.43 \\
Gammares & 0.17 \\
\hline
\end{tabular}

La consommation préférentielle d'Ephéméroptères et leur faible importance numérique dans le benthos s'expriment dans le graphique de la figure I qui met en évidence les relations inverses entre les

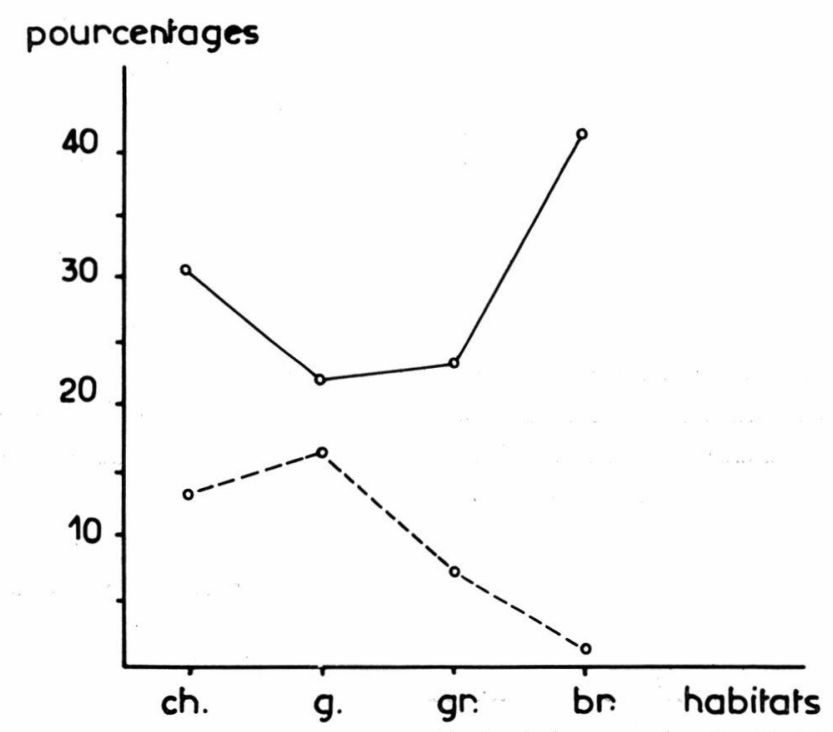

Fig. 1. - Pourcentages moyens des invertébrés prédateurs et des Ephéméroptères dans les habitats du facies lotique.

- : prédateurs ; - — : Ephéméroptères ; ch : Chiloscyphus; g : galets ; gr : graviers; br : brindilles. 
valeurs moyennes des pourcentages d'Ephéméroptères et des invertébrés prédateurs dans les principaux habitats.

\section{3. - Exemples des variations observées.}

4.3.1. - Modification du RÉGime alimentaire EN fonction de L'ÂGe OU DE LA TAILLE.

\section{- Cottus gobio}

La relation entre la taille du prédateur et la nature de la proie apparaît dans la figure 2 en accord avec les observations de Western (1968).

Les jeunes poissons capturent plus intensément des petites proies (Diptères Corynoneurinae et Orthocladiinae) alors que des individus plus âgés consomment davantage des proies de taille supérieure (Gammares, Trichoptères et Mégaloptères). Ce phénomène d'ordre

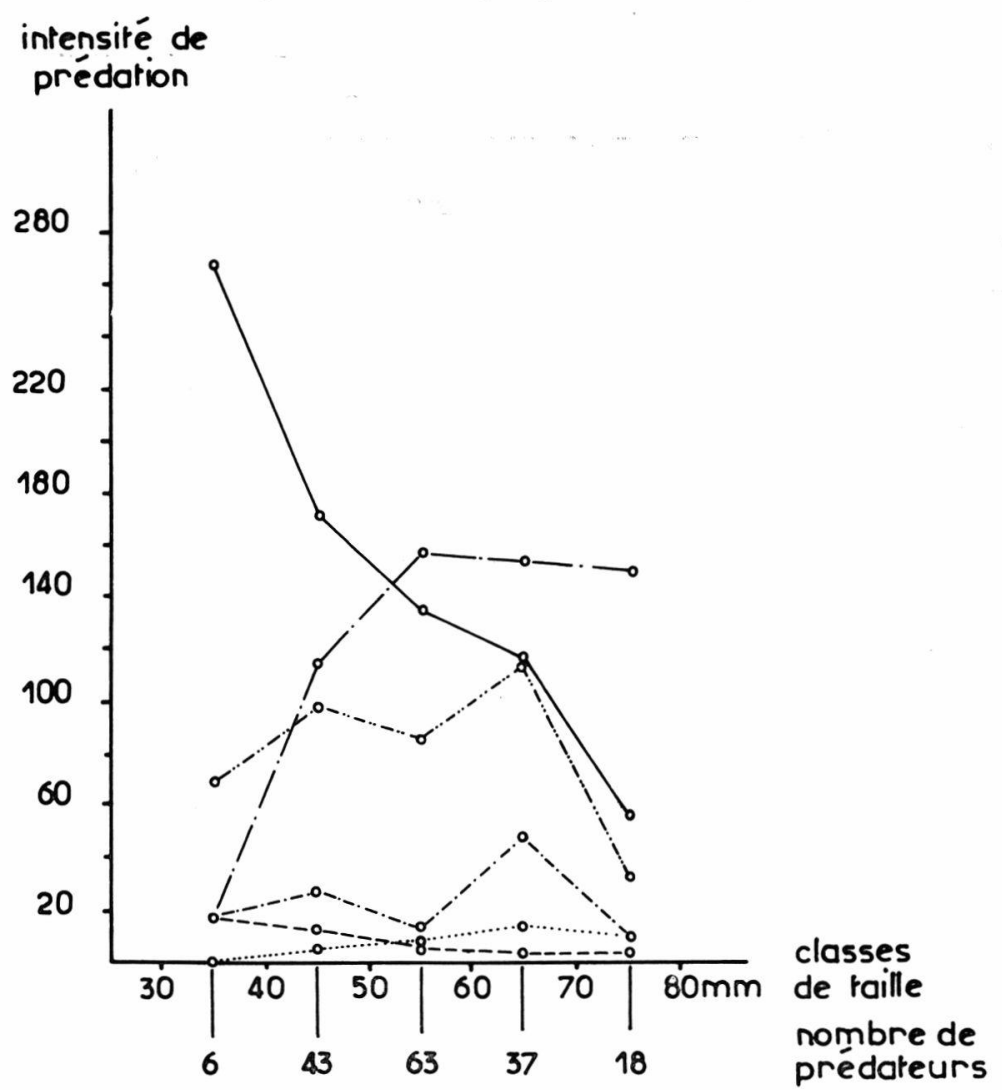

Fig. 2. - Intensité de prédation chez Cottus gobio en fonction de la taille du prédateur et de la nature de la proie.

- : Diptères ; - - - : : G. fossarum; - - : Ostracodes; - . - : Trichoptères; -.... : Baetidae; .... S. fuliginosa. 
général a été signalé par de nombreux auteurs : ANDREASson 1971 (Cottus gobio), Allen 1935 (Perca fluviatilis), Hellawell 1971 (Thymallus Thymallus), Hunt et JoNes 1972 (Salmo trutta).

- Rhyacophila septentrionis.

Phytophage puis carnassière; Décamps (1967) a précisé que le régime carnivore n'apparaît qu'aux stades 3 et 4 .

- Sialis fuliginosa.

Détritiphage à l'état de larvule puis carnassière.

4.3.2. - Modification du RÉgime alimentaire en FOnCtion de L'Époque ex. : Baetis rhodani :

Les observations ont été réalisées sur l'ensemble de la population. En janvier, l'espèce est végétarienne (diatomées), elle devient ensuite détritiphage.

La spécialisation hivernale temporaire, signalée par CHAPMAN et DENORY (1963) est sans doute liée au développement de la microflore à cette époque; ce phénomène peut constituer un exemple d'euryphagie à spécialisation temporaire selon KüHNELT (1969).

4.3.3. - Modification du RÉgime alimentaire accompagnée d'Un ChanGEMENT D'HABITAT.

ex. : Nemoura cinerea cinerea (fig. 3 ).
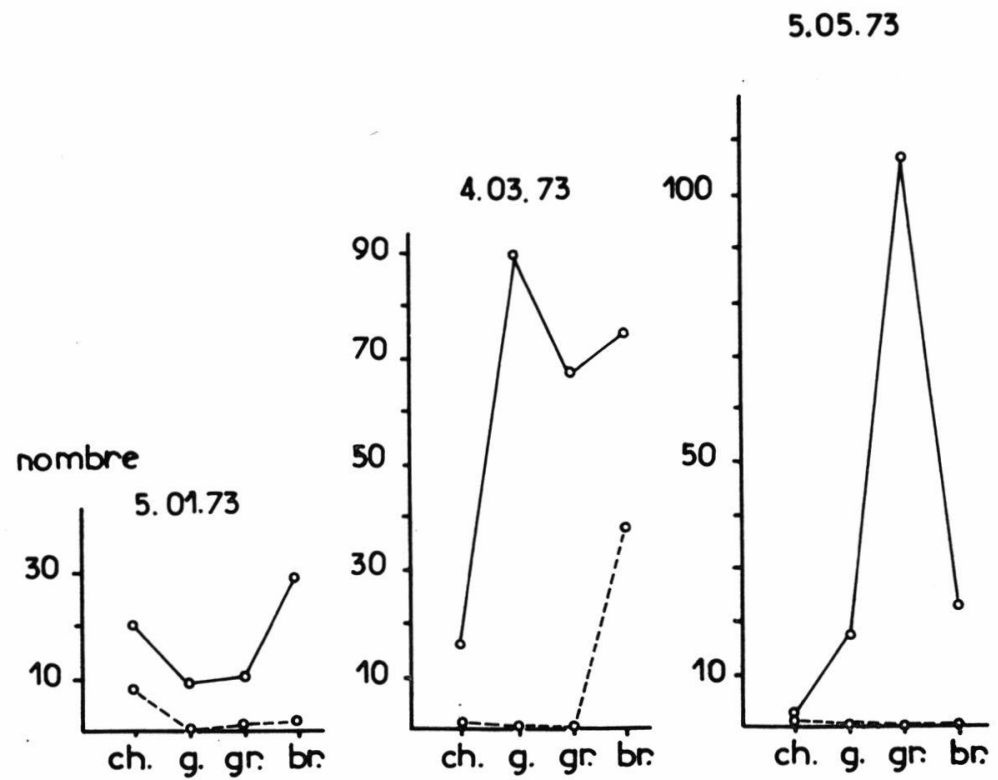

Fic. 3. - Changements d'habitats chez deux espèces d'insectes à larves aquatiques, Agapetus fuscipes et Nemoura cinerea cinerea.

ㄴ. : Agapetus tuscipes; - - : Nemoura cinerea cinerea; ch : Chiloscyphus; g : galets; gr : graviers; br : brindilles. 
Ces changements d'habitats s'effectuent au cours de la croissance des larves. En janvier, les larves $(2,5 \mathrm{~mm})$ sont phytophages; en mars la taille moyenne atteint $4,5 \mathrm{~mm}$ et les larves consomment des débris végétaux ligneux.

Les variations de la population des larves d'Agapetus fuscipes, également portées sur la figure 3, constituent un exemple de changement d'habitat à l'approche de la nymphose : les larves âgées fixent leurs fourreaux, sur les galets et graviers rivulaires, en fin de cycle.

\section{4. - Edifice trophique.}

Le dernier schéma (fig. 4) concentre les relations trophiques observées; il met en évidence l'existence d'un réseau complexe peu conforme à l'image commune de "chaîne ".

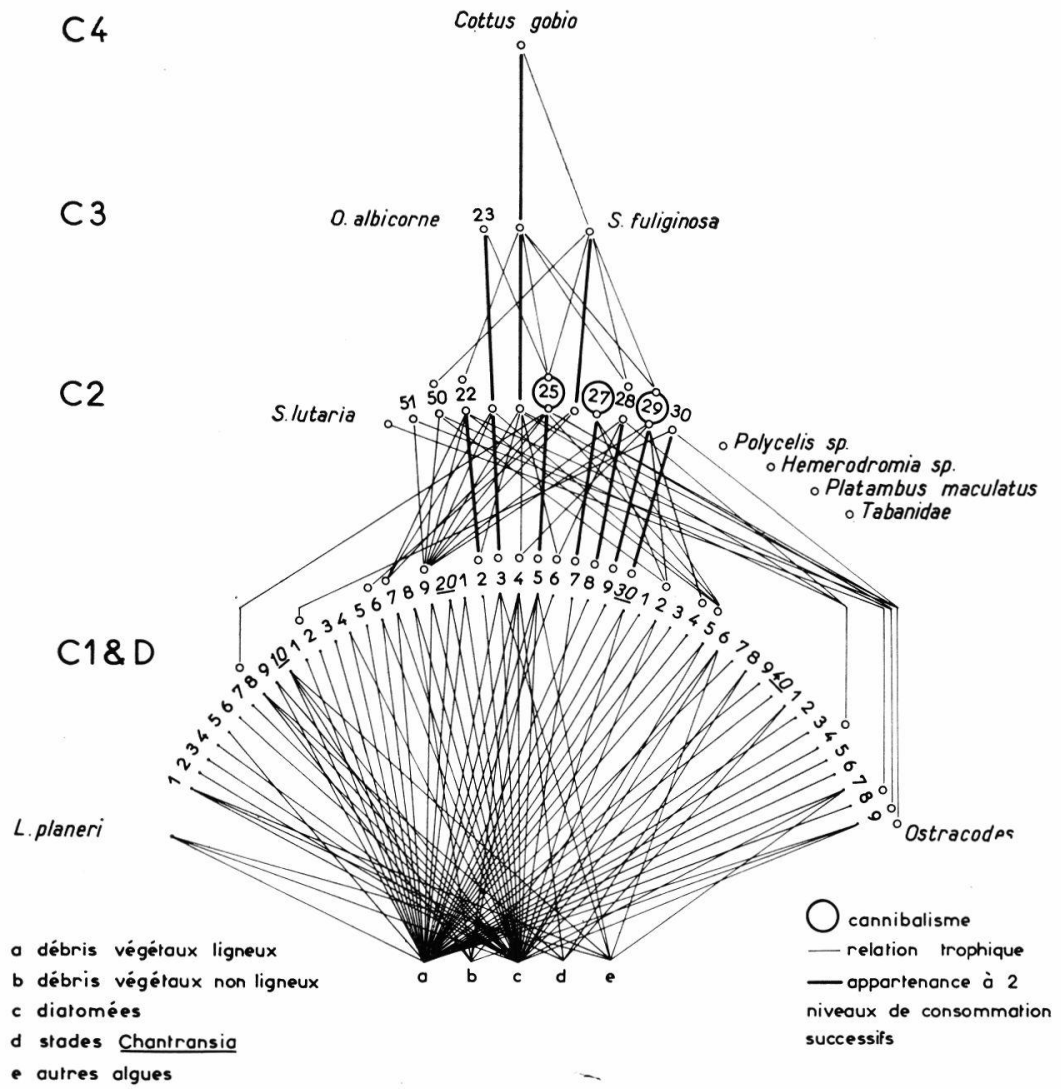

Fig. 4. - Schéma partiel des relations trophiques dans le cours supérieur des Doulonnes.

$\mathrm{a}$ : débris végétaux ligneux; $\mathrm{b}$ : débris végétaux non ligneux; $\mathrm{c}$ : diatomées; $\mathrm{d}$ : stades Chantransia; e : autres algues; $\mathrm{O}$ : cannibalisme; - : relation trophique; — : appartenance à 2 niveaux de consommation successifs. 
Les relations trophiques figurent en trait fin et pour les espèces appartenant à plusieurs niveaux de consommation (ex : Cottus gobio, Sialis fuliginosa, Odontocerum albicorne...), les passages entre niveaux successifs sont portés en trait épaissi.

Les nombres (individus et espèces) des éléments constitutifs de chaque niveau figurent dans le tableau VI ; les valeurs correspondent à l'ensemble des animaux prélevés lors des trois campagnes d'échantillonnage.

Tableau VI. - Répartition des individus et des espèces (janvier à mai) aux différents niveaux de consommation.

\begin{tabular}{ccc}
\hline $\begin{array}{c}\text { Niveau de } \\
\text { consommation }\end{array}$ & $\begin{array}{c}\text { Nombre } \\
\text { d'individus }\end{array}$ & $\begin{array}{c}\text { Nombre } \\
\text { d'espèces }\end{array}$ \\
\hline C4 & 10 & 1 \\
C3 & 95 & 3 \\
C2 & 4800 & 17 \\
C1 et D & 11700 & 50 \\
\hline
\end{tabular}

\section{5. - DISCUSSION}

\section{1. - Schéma final.}

Celui-ci constituant un bilan non limitatif des relations trophiques observées, n'implique ni la simultanéité des phénomènes, ni la cohabitation des espèces. Par ailleurs, il faudrait ajouter à ce schéma un consommateur supplémentaire, Salmo trutta fario $l$, ingérant, entre autres proies, le Chabot, donc situé au cinquième niveau de consommation.

Dans cet édifice trophique figurent des espèces dont le régime alimentaire n'a pas été précisé mais qui, considérées comme carnassières, appartiennent au deuxième niveau de consommation : Platambus maculatus (BERTRAND 1954) Hemerodromia sp. et un Tabanidae dont les larves succent les tissus de leurs victimes (VAILLANT 1954).

La complexité du réseau alimentaire, cependant relatif à un niveau écologique simple (station proche des sources), apparaît nettement ; celle-ci soulignée par de nombreux auteurs (ODuM 1959; MACAN 1963; Cummins, Koffmann et RofF 1966; Dussart 1966; Cummins 1973) met en évidence les difficultés rencontrées dans l'établissement d'un bilan, le plus précis possible, des principales voies de transferts énergétiques. Les variations du régime alimentaire en fonction de l'âge, de la taille, de la saison, ne simplifient pas le problème. 


\section{2. - Régimes alimentaires.}

- Les détritivores herbivores.

Les observations nous ont montré qu'il n'existe, en fait, aucune espèce strictement détritivore, les débris végétaux altérés sont absorbés avec une certaine quantité de diatomées (le plus fréquemment) ou d'autres algues dont l'abondance dépend du lieu d'alimentation; comme l'a constaté Hynes (1970), ce mélange de débris végétaux, frustules de diatomées et particules minérales, constitue le bol alimentaire le plus fréquent, chez les invertébrés aquatiques.

- Régime alimentaire et mode de vie.

Comme le font remarquer Lavandier et Dumas (1971), " si des relations entre habitudes nutritives et microdistribution peuvent être établies pour les consommateurs primaires stricts (racleurs de substrat selon Décamps 1968), ces relations sont beaucoup moins nettes pour les détritivores, omnivores ou carnassiers ».

Toutefois, dans le cas d'une alimentation carnée, les coefficients de sélection, appliqués au niveau des espèces, peuvent apporter de précieuses informations quant aux probabilités de rencontre, de cohabitation entre consommateur et consommé (ex. Cottus gobio et Sialis fuliginosa).

- Le cannibalisme.

A défaut d'éléments de comparaison (pour l'instant) quant à l'occurrence du cannibalisme, mentionnons que celui-ci est apparu pour des densités de l'ordre de 5000 à 10000 individus $/ \mathrm{m}^{2}$ chez Gammarus fossarum, 3000 individus $/ \mathrm{m}^{2}$ chez Apsectrotanypus trifascipennis (Tanypodinae), et de 1000 individus $/ \mathrm{m}^{2}$ chez Procladius sagittalis (Tanypodinae).

L'existence d'un régime omnivore chez certains Tanypodinae (RoBACK 1969) et de cas de cannibalisme sont à rapprocher d'observations effectuées par FAHY (1972) qui, ayant comparé la nutrition d'une dizaine d'espèces carnivores dans deux situations aquatiques extrêmes ( « in a poor trophic headwater type stream " et « in a richer, lower catchment type situation"), note que dans des conditions d'oligotrophie certaines espèces complètent leur régime avec du matériel végétal; puis la prédation s'accentue et le cannibalisme apparaît.

\section{3. - Régime alimentaire et niche écologique.}

A la définition «fonctionnelle " de la niche écologique en tant que "place d'un animal dans l'environnement biotique, ses relations avec la nourriture et avec les ennemis " (ElTON in KendeIgH 1961), Odum (1959) ajoute la nécessité de considérer l'effet des organismes sur l'environnement " abiotique ", cette opinion ayant également été retenue par l'un de nous (Verneaux 1973). 
Ceci peut être partiellement retrouvé dans le cadre de l'analyse des régimes alimentaires et des modes de vie.

- Phytophage : l'espèce peut intervenir sur le développement et l'abondance de la couverture biologique primaire; dans cette évantualité elle peut agir également sur le transport, la régulation des peuplements et la dispersion des algues consommées (Solon et STEWART 1971).

- Détritivore : elle peut influencer l'évolution des sédiments (Lampetra planeri, Ephemera danica, de nombreux Oligochètes et Diptères); on note le même phénomène dans l'évolution des litières de feuilles mortes (Gammaridae, Sericostomatidae et Limnephilidae, Chironomidae), bien que dans ce dernier exemple, il semble que la microflore (bactéries et champignons) soit également responsable de l'évolution et constitue ainsi la source énergétique principale des détritivores (Mathews et Kowalczewski 1969; Hynes 1970).

- Carnivore : elle peut régler la densité des populations d'espèces prolifiques et aisément disponibles comme Gammarus fossarum et Baetis rhodani.

Par ailleurs, les variations enregistrées au niveau de l'habitat ou du régime alimentaire de certaines espèces, témoignent de l'existence d'aspects différents de la niche écologique au cours de leur développement. Dussart (1970) a regroupé sous le terme "d'écophase", l'ensemble des individus, en général d'une seule espèce, "qui occupent la même niche pendant le même temps "; il ajoute que «l'écophase " constitue l'unité élémentaire en écologie et qu'elle doit être étudiée en tant que telle. On remarque que cette notion est surtout mise en évidence lorsque l'on considère le rôle joué, la fonction au lieu des acteurs, comme une partie de la niche écologique globale d'une espèce donnée.

Certaines écophases ont été pressenties dans ce travail lors de l'observation de changements d'habitats, de modifications de régime alimentaire au cours du développement. Demeurent à préciser dans une large mesure, leurs identités réelles au cours de l'analyse de situations variées ainsi que les passages de l'une à l'autre à l'occasion du développement des espèces et des variations des complexes mésologiques.

\section{TRAVAUX CITÉS}

Allen (K. R.). 1935. - The food and migration of Perch (Perca fluviatilis) in Windermere. J. Anim. Ecol., 4 : 264-273.

AlleN (K. R.). 1941. - Studies on the biology of the early stages of the Salmon (Salmo salar) -2 . Feeding habits. J. Anim. Ecol., $10: 47-76$.

ANDREasson (S.T.). 1971. - Feeding habits of a sculpin (Cottus gobio L. Pisces) population. Rep. Inst. Freshw. Res. Drottningholm. 51: 5-30. 
Bertrand (H.). 1954. - Les Insectes Aquatiques d'Europe. Lechevalier éd., Paris. I : 556 p., II : $547 \mathrm{p}$.

ChaPMAN (D. W.) et DEMORY (R. L.). 1963. - Seasonal changes in the food ingested by aquatic insect larvae and nymphs in two oregon streams. Ecol. 44 (1) : $140-146$.

Cummins (K. W.), Koffmann (W. P.) et RofF (P. A.). 1966. - Trophic relations in a small woodland stream. Verh. Int. Verein,. Limnol., $16: 627-638$.

Cummins (K. W.). 1973. - Trophic relations of aquatic insects. Ann. Rev. Entomol. $18: 183-206$.

Decamps (H.). 1967. - Ecologie des Trichoptères de la vallée d'Aure (HtesPyrénées) Annls Limonol., 3 (3) : 399-577.

Decamps (H.). 1968. - Vicariances chez les Trichoptères des Pyrénées. Annls Limnol., 4 (1) : 1-50.

Dumont (B.). 1973. - Edifice trophique partiel du cours supérieur d'un ruisseau forestier (les Doulonnes, massif du Jura). DEA Fac. Sci. Besançon, 17 p.

Dussart (B.) 1966. - Limnologie. L'étude des eaux continentales. Gauthier-Villars éd., Paris, 676 p.

Dussart (B.) 1970. - Quelques problèmes d'écologie. Bull. A.P.B.G., 3 : 245-257.

FAHY (E). 1972. - The feeding behaviour of some lotic insects in two streams of differing detrital content. J. Zool. Lond., 167 : 337-350.

Feldman (J.). 1963. - Les algues. Précis Botan., Masson éd., Paris : 83-249.

GRIFFITHS (D.). 1970. - Observations on the food of dragongly nymphs, from a bog water in North Norway. Entomologist mon. mag., 106 : 31-47.

HanNa (H. M.). 1957. - A study of growth and feeding habits of the larvae of four species of caddisflies. Proc. Roy. Entomol. Soc. Lond., 32 : 139-146.

Hellawell (J.M.). 1971. - The food of the Grayling (Thymallus thymallus) of the river lugg., Herefordshire. J. Fish. Biol. : 187-197.

HunT (P.C.) et Jones (J.W.). 1972. - The food of brown trout in Llyn Alaw, Anglesy, North Wales. J. Fish. Biol., $4: 182-205$.

HyNes (H. B. N.). 1970. - The ecology of running waters. Liverpool Univ. Press éd. : $555 \mathrm{p}$.

Illies et Botosaneanu (L.). 1963. - Problèmes et méthodes de la classification et de la zonation écologique des eaux courantes considérées surtout du point de vue faunistique. Mitt. internat. Verein. Limnol. $12: 1-57$.

Jenkins (T. M.), Feldmeth (C. R.) et Elliot (G. V.). 1970. - Feeding of Rainbow Trout (Salmo gairdneri) in relation to abundance of drifting invertebrate in a mountain stream. J. Fish. Res. Canada, 27 (12) : 2356-2361.

JoNes (J. R. E.). 1950. - A further ecological study of the river Rheidol. The food of the common insects of the main stream. J. Anim. Ecol. 19: 159-174.

Keindeigh (C. H.). 1961. - Animal ecology. Englewood Cliff. N.J.: 468 p.

Koslucher (D. G.) et Minshall (G.W.). 1973. - Food habits of some benthic invertebrates in a northurn cool-desert stream (Deep creek, Curlew Valley Idako-Utah. Trans. Amer. Micoros. Soc., 92 (3) : 441-452.

Kuhnelt (W.). 1969. - Ecologie générale. Masson éd., Paris : 359 p.

Ladle (M.), Bass (J. A. B.) et Jenkins (W. R.). 1972. - Studies on production and food consumption by larval simuliidae (Diptera) of a Chalk stream. Hydrobiol., 39 (3) : 429-448.

Lavandier (P.) et Dumas (J.). 1971. - Microrépartition de quelques espèces d'invertébrés benthiques dans les ruisseaux des Pyrénées Centrales. Annls Limnol., 7 (1) : 7-23.

Macan (T. T.). 1963. - Freshwater Ecology. Longmans, Green and Co. ed., London : $338 \mathrm{p}$.

MADSEN (B. L.). 1974. - A note on the food of Amphinemura sulcicollis (Plecoptera). Hydrobiol., 45 (2-3) : 169-175.

ManN (K. H.). 1969. - The dynamics of aquatic ecosystems. Advances in ecological Research. Academic Press, $6: 1-81$.

Mathews (C. P.) et KowalczewsKi (A.). 1969. - The disappearance of leaf litter and its contribution to production in the river Thames. J. Ecol., 57 : 543-552.

Odum (E. P.). 1959. - Fondamentals of Ecology. W.B. Saunders Cy ed., Philadelphia : $546 \mathrm{p}$ 
Richardson (J.W.) et Gaufin (A. R.). 1971. - Food habits of some western stonefly nymphs. Trans. Amer. Entomol. Soc., 97 : 91-121.

Roback (S. S.). 1969. - Notes on the food of Tanypodinae larvae. Entomol. News, $80: 13-18$.

SiefFerT (R. E.). 1972. - First food of larval Yellow Pech, White Sucker, Bluegill, Emerald Shiner and Rainbow Smelt. Trans. Amer. Fish Soc., 101 (2) : 219-225.

Slack (H.D.). 1936. - The food caddish larvae. J. Anim. Ecol., 5 : 105-115.

Solom (B. M.) et STEWART (K. W.). 1971. - Dispersal of algae and protozoa via the alimentary tract of selected aquatic insects. Environ. Entomol., $1: 309-314$.

Valllant (F.). 1954. - Recherches sur la faune madicole de France, de Corse et d'Afrique du Sud. Th. Sci. Nat., Paris : 258 p.

VerneauX (J.). 1968. - Contribution à l'étude d'une petite rivière de plaine : les Doulonnes (Jura) - Référence particulière aux Diptères Chironomides - Remarques écologiques : Ann. Sci. Univ. Besançon Zool., $4: 1-12$.

VERNEAUX (J.). 1973. - Recherches écologiques sur le réseau hydrographique du Doubs. Essai de biotypologie. Thèse Sci. Nat. Fac. Sc., Besançon, C.N.R.S., 8823. Ann. Sci. Univ. Besançon, Zool., Physiol, Biol. Anim., 3 (9) : $260 \mathrm{p}$.

WESTERN (J.R. H.). 1968. - Studies on the diet, feeding mechanism and alimentary tract in two closely related Teleosts, the freshwater Cottus gobio L. and the marine Parenophrys bubalis Euphrasen. Act. Zool., $50: 185-205$. 